\title{
A SURVEY OF REACTOR-NOISE TIME-ANALYSIS METHODS
}

AUGUST 1968

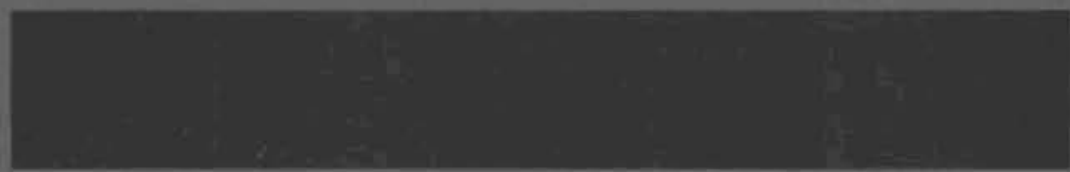

\section{AEC RESEARCH \& DEVELOPMENT REPORT}




\section{LEGAL NOTICE}

This report was prepared as an account of Government sponsored work. Neither the United States, nor the Commission, nor any person acting on behalf of the Commission:

A. Makes any warranty or representation, expressed or implied, with respect to the accuracy, completeness, or usefulness of the information contained in this report, or that the use of any information, apparatus, method, or process disclosed in this report may not infringe privately owned rights; or

B. Assumes any liabilities with respect to the use of, or for damages resulting from the use of any information, apparatus, method, or process disclosed in this report.

As used in the above, "person acting on behalf of the Commission" includes any employee or contractor of the Commission, or employee of such contractor, to the extent that such employee or contractor of the Commission, or employee of such contractor prepares, disseminates, or provides access to, any information pursuant to his employment or contract with the Commission, or his employment with such contractor.

\section{PACIFIC NORTHWEST LABORATORY \\ RICHLAND, WASHINGTON \\ operated by}

BATTELLE MEMORIAL INSTITUTE

for the

UNITED STATES ATOMIC ENERGY COMMISSION UNDER CONTRACT AT(45-1)-1830 


\title{
33679000611774
}

\author{
BNWL- 875 \\ UC-80 Reactor Technology \\ Special Distribution
}

A Survey of Reactor-Noise Time-Analysis Methods

\begin{abstract}
N. Pacilio (1)(2)
Reactor Experiments Section

Reactor Physics Department
\end{abstract}

\begin{abstract}
August 1968
PACIFIC NORTHWEST LABORATORY

RICHLAND, WASHINGTON
\end{abstract}

(1) Exchange Scientist from Comitato Nazionale Energia Nucleare (CNEN), Centro Studi Nucleari della Casaccia, Rome, Italy, under cooperative agreement between USAEC and CNEN.

(2) Present address: Reactor Physics Division, Argonne National Laboratory, Argonne, Illinois. 
Printed in the United States of America Available from

Clearinghouse for Federal Scientific and Technical Information National Bureau of Standards, U.S. Department of Commerce Springfield, Virginia 22151

Price: Printed Copy $\$ 3.00$; Microfiche $\$ 0.65$ 
Premise $\quad$ iv

Introduction $\quad 1$

General Considerations on Reactor-Noise Time-Analysis Methods 4

Section 1: Pulse-Time Correlation Analysis Methods 8

Rossi-Alpha 8

$\begin{array}{ll}\text { Endogenous Pulsed Source } & 10\end{array}$

$\begin{array}{ll}\text { Auto and/or Cross Correlation } & 13\end{array}$

Section 2: Analysis of Moments of Counting Distributions 16

Feynman Variance Method 16

Expected Number of Correlated Neutron Pairs Method 20

$\begin{array}{ll}\text { Bennett Variance Method } & 22\end{array}$

Third Order Analysis Method 24

Covariance Analysis Method 26

Section 3: Differentiae Analysis of Counting Distributions 28

Zero-Count Probability Method 28

Polya Model Method 30

Other Related Probability Methods: 32

Zero-Counc to One-Count Probability Ratio 32

Furubashi-Izumi Probability Method $\quad 32$

Even-and/or Odd-Count Probability 33

Time Interval from a Random Origin Method 36

Pulse-to-Pulse Time Interval Method 38

Section 4: Correlation of the Signs of Fluctuations about a 41

Mean Value

Polarity Auto-and/or Cross-Correlation Method 41

Covariance Method via Polarity Detection 43

References 
PREMISE

The present paper has been condensed from a series of seminars given by the author, during the first part of 1968, at the Department of Nuclear Engineering, University of California, Berkeley and at the Physics and Engineering Division, Battelle Northwest Laboratories, Richland, Washington.

The short survey of the included methods is accompanied by a limited number of references, i.e. two for each of the reported techniques. The reason of such an approach is that of giving only some points of departure for a further insight to the theoretical and experimental problems connected to these reactor-noise analysis techniques.

A more exhaustive list of references, together with a more extensive treatment of the subject, will be given in a state-of-the-art monograph that is going to be prepared by the same author and published at the end of this year under the auspices of the U. S. Atomic Energy Commission. 
BNWL -875

1

SURVEY OF REACTOR-NOISE TIME-ANALYSIS METHODS

N. Pacilio

\section{INTRODUCTION}

Reactor-noise analysis techniques were originally based on sampling the neutron population in the time domain. This way, they were restricted to the zero power reactor class, because of the physical limitation in the repetition rate of the pulse counters employed in such measurements. Furthermore these methods were all detection efficiency limited ( only with the exception of the Rossi-alpha procedure ) and so actually unsuitable for fast reactor analysis.

The extension of these types of measurements - or, better, a kind of Fourier transform of them - to the frequency domain opened the opportunity of realizing a meaningful noise analysis even in non-zero power reactors through the use of current chambers, band-pass filters and the advantageous application of the auto and cross correlation techniques for the elaboration of data. A frequency range wider than that stimulated by spontaneous fluctuations and/or a larger noise amplitude in certain frequency regions were sometimes excited by the external modulation of neutron waveforms to insert into the system.

However the frequency domain approach featured some inconveniences, i.e. the necessity of special equipment, the inapplicability to fast reactors, the time consuming data reduction, the fact that the reactor trans- 
fer function obtained by analog techniques is more difficult and less precisely interpreted in terms of roll-off frequencies than a time decay can be in terms of multiple exponential constants. The latter statement is particularly critical if reflected and multizone reactors are under observation, since the described situation needs an accurate analysis in these cases.

These inconveniences and a particular attention dedicated to reactor noise physics by a constantly increasing number of authors and laboratories have brought the spot again on the time-analysis theory and experiments and have given a vast contribution in terms of new methods and suitable refinements of the old ones.

Thus, the most recent trend of noise analysis is oriented to the consideration of a discrete noise field to be sampled in the time domain. This return to the study of individual pulses must be essentially intended as an extension of the original approach, motivated by new theoretical and technological opportunities, which now allow a profitable application of noise techniques to any type of reactor.

Now that the fundamental limitations of this approach seem to be circumvented, the horizon of application of the time-analysis methods becomes enlarged and seriously competitive with that of the frequency analysis, whose developments in the last years have been less impressive, at least in regard to reactor kinetic parameter measurement methods. 
The main elements of the new situation are:

1) the improved characteristics of analog to digital converters, which realize the quantization of a signal even when a pulse counter cannot be used directly in 1oco, viz. in all systems from plutonium assemblies to power reactors:

2) the time cross correlation techniques between two detectors which bypass the limitation of low efficiency in fast reactors and give the only essential term of the noise, that containing the required information about the decay eigenvalues;

3) the possibility of using on-line digital computers, instead of time-analyzers, which allow a more compact and rapid treatment of data, as well as a higher sampling rate and an almost unlimited accumulation of data together with a quasi-continuous check of the correctness of the experiment:

4) the development of new techniques in which only the parity and/or the polarity of the involved variables is needed to determine the time correlations and, therefore, the reactor parameters. Our presentation will be limited to a short description of all the techniques related and/or applied to the reactor fluctuations in the time domain. 
BNWL -875

4

GENERAL CONSIDERATIONS ON REACTOR-NOISE TIME-ANALYSIS METHODS

The first fundamental assumption of all the methods considered is that the reactor is in steady state operating conditions, i.e. critical or subcritical in equilibrium with a uniform source. This may not be strictly indispensable for some procedures but to make such an assumption represents a useful scheme.

The neutron population is observed through a suitable decector whose output is either integrated or simply counced in the time dornain; the neutron energy is usualiy disregarded. The instrumentation employed for these measurements can be the common neutron monitoring apparatus of the reactor (pulse counters and current chambers) with the exception of special căses in which high detection eificiencies are needed and some in-core instivitientation must be added.

A conmon feature among the procedures is that all the techniques are based on observing the prompt neutron chains which are dying away because of the prompt subcricical conaitions. Their decay constants determine the order of magnitude of the times involved in such analysis; they are approxImateì

$$
\begin{aligned}
100 \mathrm{msec} & \text { for } \mathrm{D}_{2} \mathrm{O} \text { and graphite reactors } \\
10 \mathrm{msec} & \text { for } \mathrm{H}_{2} \mathrm{O} \text { and organic reactors } \\
1 \mathrm{insec} & \text { for pure plutonium reactors } \\
1-10 \mathrm{sec} & \text { for fast reactors }
\end{aligned}
$$

Since all the rechniques have a statistical derivation, the whole analysis is not based on single events, 1.e. time separation between two nelitrons, but on the observation and storing of a large amount of data. 
Two appro aches are essentially possible. One of them is strictly a time analysis since it is based on the localization of the arriving neutrons on a time axis; the second one is of integral type and consists in counting the number of neutrons detected in fixed time intervals, collecting the data as a time sequence $n_{j}(t+j \Delta t)$ or suitable reductions of $i t$. The symbol denotes the number of pulses occurred (or the integrated output) in a time interval $t+j \Delta t \longmapsto t+(j+1) \Delta t, t$ being an instant in reactor Iife, $\Delta t$ the time interval adopted and $j$ an integer number $(j=0,1$, $2, \ldots, N)$ which characterizes the sequence.

Four different types of procedure can be distinguished according to how these outputs are manipulated. They yield a total of fifteen methods and can be divided as follows:

1) methods based on time correlation analysis among the pulses, in which the various correlation functions can be calculated off-1ine or approximated by a suitable arrangement of the measuring apparatus (delayed coincidence system, time analyzer or multiscaler with repetitive cycles). They include

- the Rossi-alpha method

- the endogenous 'pulsed source' method

- the auto and/or cross correlation method

- the time interval from a random origin method*

- the pulse to pulse time interval method*

* Note:Although belonging to the time analysis category, these two methods will be attached to group 3 for better understanding. 
2) methods based on analysis of the moments of the distribution $\left\{n_{j}\right\}$ in which a calculation limited to the second (or third order) is generally sufficient in giving the desired information. They include

- the Feynman variance method

- the expected number of correlated neutron pairs method

- the Bennett variance method

- the third order analysis method

- the covariance mechod

3) methods based on differential analysis of the distribution $\left\{\mathrm{n}_{\mathrm{j}}\right\}$ in which the experimental data are compared with some theoretical probability profiles in order to find the fitting parameters. They include

- the zero-count probability method

- Lhe Polya model method

- some incermediate methods (the zero-count to one-count probability ratio method, the Furuhashi-Izumi probability methư,

- the even-and/or odd-count probability method

4) methods based on correlation analysis among the signs of the tluctuations of $\mathrm{n}_{j}$ around $i t s$ mean value, in which the interpretation is in terms uf the autocorrelation function for one detector output and in terms of cross correlation and/or covariance of the data for a two detector output. They include

- the polarity auto and/or cross correlation method

- the covariance method via polarity detection 
Al1 the described techniques provide the determination of time behaviour parameters (e.g. the prompt neutron decay constant $\alpha$ ) as well as that of static parameters (e.g. reactivity, power) replacing and extending the now obsolete original reactor physics procedures born together with the first multiplying assemblies.

In fact, for a nuclear system, the prompt period at criticality, viz. the ratio of the prompt neutron lifetime to the effective total fraction of delayed neutrons, is one of the most important parameters for comparison between theory and experiments, since it allows basic data concerning cross-section libraries, spectral distributions and discretization into groups, importance function etc. to be checked directly with measurements results. Furthermore a comparison between calculated and observed $\alpha$ offers valid information beyond and beside the critical experiment. In fact, for subcritical systems, the parameter $\alpha$ is at least as much expressive as reactivity with the difference that $\alpha$ is observable while reactivity is not. This suggests a new approach to all the reactivity related coefficient measurements, i.e. fuel, rods, temperature, moderator level if any, which is extendable to highly subcritical systems unlike all other reactlvity-based procedures.

All the mathematical developments featured in this survey are based on a bare monoenergetic diffusion-theory model: of ten the prompt neutron kinetics approximation is adopted. 
BNWL -875

Section 1: Pulse-Time Correlation Analysis Methods

THE ROSSI-ALPHA METHOD

The Rossi-alpha method, ${ }^{(1,2)}$ named after Bruno Rossi who suggested the experiment during the early days of Los Alamos, is essentially based on the observation of the prompt neutron decay, viz. the time distribution of counts following a certain triggering pulse due to a randomly chosen 'first' neutron detection.

Counts uncorrelated with the initial one are likely to occur any time after the triggering event and therefore have a constant probability of detection. Counts correlated with the initial one, i.e. neutrons be-longing to the same fission chain, exhibit a time-decreasing probability of occurrence because of the subcritical prompt multiplication factor.

If we call $P(t) d t$ the probability that after a pulse at time $t=0$, a pulse arrives between $t$ and $t+d t$, we have that

$$
P(t) d t=A d t+B e^{-\alpha t} d t
$$

where $A$ is the counting rate, $B$ is a term depending on reactor reactivity and detector efficiency and $\alpha$ is the prompt neutron decay eigenvalue defined as

$$
\alpha=\frac{1-k_{p}}{l}-=\frac{1}{l} \frac{\beta-R}{1-R}=\frac{\beta}{\Lambda}(1-\$)
$$

The symbols denote the prompt multiplication factor $\left(k_{p}\right)$, the prompt neutron lifetime $(l)$ and generation time $(\Lambda)$, the effective total frac-. tion of delayed neutrons $(B)$, the reactivity $(R)$ and the reactivity in doliar units $(\$)$. 
BNWL-875

Therefore an investigation of the time behaviour of this detection probability yields a determination of one or more reactor kinetic characteristics, i.e. the prompt reactor period, the ratio of the effective total fraction of delayed neutrons to the prompt neutron lifetime, the reactivity of the system, the power level and so forth. This type of experiment can be used as valid alternative of the pulsed neutron measurements, mainly at criticality and in slightly subcritical conditions, where the former procedure becomes experimentally unreliable.

The technique is profitab1y applied to fast systems under low-power operating conditions. It can be extended-although with smaller convenience and efficiency-to thermal reactors if proper modifications are made. It cannot be used in non-zero power reactors (from plutonium assemblies on) and it becomes impracticable when the spontaneous neutron source exceeds $1 / 5$ neutron per prompt neutron lifetime.

The original instrumentation employed for such an experiment was a system of ten delayed coincidences between every detected neutron pulse and all the following ones. The reactor power level and the detection efficiency were set at appropriately low values in order to prevent significant overlapping of the neutron chain detected.

Since the original experiment, the Rossi-alpha designation has been quite often applied to several investigations more or less related to the original one. They could still be interpreted in terms of the formalism of Eq. (1) if suitable modifications of practical and interpretative nature are operated, case by case. 
They are due to the adoption of

1) two detectors; one gives the triggering pulses, the other gives the pulses to be time-analyzed

2) a multichannel time-analyzer instead of the delayed coincidence system; it can be used in such a way that more than one pulse per sweep can be recorded or in the so called stop-watch technique. Here the time analysis is triggered by one detector and stopped by the first arriving pulse from the second detector

3) a multiscaler, especially for thermal reactor experiments. Measuring apparati as 2) and 3) lead to experiments immediately seen as different from the original Rossi-alpha procedure. They in fact neglect part of the correlations among neutron pulses and, therefore, underestimate the amplitude B of Eq. (1). Fortunately the value of $\alpha$ is not biased by such a modified approach, but it may happen that $B$ is so damped that the observed profile is completely flat and a cannot be derived.

\section{THE ENDOGENOUS 'PULSED SOURCE' METHOD}

The method $(3,4)$ is based on observation of the time distribution of counts following a certain triggering pulse which occurs if the spontaneous fluctuations of the reactor power reach a preselected value above the mean level.

Since the inherent neutron fluctuations can be described in terms of an equivalent pulsed source with variable intensity and repetition rate the technique has been named the endogenous 'pulsed source' method (eps). 
If the appearance of spontaneous bursts can be essentially considered as random and due to sudden fission rate variations, the proliferation and decay of the bursts from the attained peak occur according to the reactor kinetic decay eigenvalue $\alpha$.

In fact, if we call $n(t)$ the neutron density, we have that after each spontaneous burst

$$
n(t)=n_{0} e^{-\alpha t}+\bar{n}
$$

where $\bar{n}$ is the mean value of counts (i.e., A $\Delta t, \Delta t$ being the time-width of each multiscaler channel) and $n_{0} / \bar{n}$ is the signal to background ratio, i.e., the relative amplitude of the analyzed burst above the mean level and with respect to it.

This type of technique can be considered as intermediate between the purely statistical Rossi-alpha experiment and the classical pulsed-source technique, showing similarities to both of them in the experimental procedure and in the interpretation of data and sharing some of the advantages of both.

The basic improvement, due to the preselection device, appears essentially in the increased signal/background ratio since only decay chains of conspicuous amplitude are time-analyzed an, therefore, flat or almost flat runs are avoided.

Thus, the eps technique allows statistical experiments of Rossi- $\alpha$ type to be performed in thermal reactors for reasonably acceptable measurement times while the conventional Rossi-alpha methods is widely 
known as prohibitively time consuming in such systems. The eps experiment features also practical advantages when compared to pulsed neutron measurements, since the expensive apparatus of the pulsed source is no longer necessary. Furthermore the setting up of the experiment and the reactor management are greatly simplified without the presence of the commonly bulky pulsed source. However the latter statements are a preferential feature of all the neutron noise analysis experiments. The technique can be possibly extended with similar benefits and improvements to fast reactors by applying a suitable preselection device to the triggering detector used for Rossi-alpha measurements.

The preselection and trigger device constitutes the most important part of the whole measuring apparatus, whose other elements are identical to those employed in the modified Rossi-alpha experiment in thermal reactors, i.e. a single detector and a multiscaler.

Four different types of burst monitors have been used. They are

1) a fast ratemeter, which is adjusted to give an output signal as soon as a certain preset value of the counting-rate is reached,

2) a compensated pair of two counting-rate meters, with highly different time constants. The occurrence of a burst is clearly detected by the fast ratemeter and ignored by the slow one, so that the uncompensated signal can trigger the multiscaler,

3) a digital preselector set at the desired $n_{0}, \bar{n}$ value in order to give an output pulse every time that a count equal or greater than $n_{0}+\bar{n}$ is recorded in a time interval $\Delta t$, 
4) a coincidence system, which operates as follows. A pulse from the neutron detector opens a coincidence gate of a certain duration depending on the instantaneous repetition rate one wants to select (i.e. amplitude of the rising spontaneous burst). If a second pulse comes within this time intervel, another coincidence gate is opened waiting for a third pulse. The arrival of this pulse-within the fixed coincidence gate - makes the circuit give an output which triggers the multiscaler. The number of the three pulses divided by the entire duration of the two coincidence gates gives the minimum repetition rate selected. Further coincidence stages can be added in order to better discriminate the presence of the bursts against the mean counting level.

\section{THE AUTO/OR CROSS CORRELATION METHOD}

As we have stated in the introduction, a time behaviour analysis of a certain nuclear system can be realized by recording the counting rates $\mathrm{n}_{j}(t+j \Delta t)$ ordered in their time sequence according to the increasing values of the integer number $i$.

Assume in particular that the quantities $n(t)$ and $n(t+\tau)$ are under observation, for one or more values of the $\operatorname{lag} \tau$. If the phenomena involved are random and stationary, the average product over the whole ensemble of pairs $n(t)$ and $n(t+\tau)$, viz.

$$
\langle\mathrm{n}(\mathrm{t}) \mathrm{n}(\mathrm{t}+\tau)>
$$

must clearly be identical for any value of $\tau$.

On the contrary, if a certain time evolution takes place in the system, the same product contains information about its behaviour from the time $t$ to the time $t+\tau$ and, therefore, assumes a different 
magnitude according to the value of $\tau$ involved.

An experimental procedure based on this approach is one of the many tools which go under the name of methods of correlaticn analysis: they have been applied to various systems to measure their dynamic characteristics.

In particular, the previously mentioned averaged product is called auto correlation function if the two variables are the same detector output, cross correlation function if the detectors are two.

When applied to nuclear reactors, the general formulation of the problem is useful to set a fundamental theoretical basis for all the types of experiments described in the present paper, although some of them were originally proposed and realized on the basis of more heuristic treatments of some specific problems.

For one or two neutron detectors, $(5,6)$ placed inside or near a nuclear assembly, the auto or cross correlation function of the outputs is given by

$$
\begin{aligned}
& C_{11}(t)=A^{2}+A B e^{-\alpha t} \quad t>0^{+} \\
& C_{12}(t)=A_{1} A_{2}+A_{1} B_{2} e^{-\alpha t}
\end{aligned}
$$

A comparison between Eq. (1) and (3) shows a substantial identity apart from the proportionality factor A which indicates the neutron counting rate. The similarity between the two final formulae reflects the essentially identical phenomenology and the analogous data processing although different experimental apparati and elaboration of data are apparently employed. 
The similarity can be explained if a deeper insight into the correlation algorithm is undertaken.

Assume that a string of data has been recorded: its binary content can be 0 (no pulse) or 1 (one pulse) for each time interval. The calculation of the correlation function is equivalent to comparing the original string with the same string shifted back a number of intervals, whose sum is equal to the lag $\tau$, and to count the number of channels with a simultaneous content equal to 1 on both strings.

It is immediately clear that the described algorithm is physically equivalent to counting the number of delayed coincidences among the two strings for a delay-time given by the lag $\tau$. It is also equivalent to a determination of the time interval among every pulse of the original string and all the following in other strings and/or to counting the number of pairs which are separated by a time interval $\tau$.

This type of analysis is performed by the delayed coincidence system; in fact, it measures the cross correlation function of the neutron counting rates simultaneously for ten successive delay-times. The present discussion clarifies also how the original physical meaning of the Rossi-alpha - actually a real correlation analysis procedure - is distorted when time-analyzers are employed as measuring devices and how much information on the time sequence of pulses is lost because of the adoption of this discontinuous logic. 
Although the auto-and cross-correlation methods are the most straightforward and direct to be understood under the viewpoint of the physical meaning involved, the practical realization of such experiments requires an off-1ine, longer and more elaborated treatment of the collected data when compared with the classical Rossi-alpha experiment whose outpui is already in its definitive form.

Section 2: Analysis of Moments of Counting Distributions THE FEYNMAN VARIANCE METHOD

The Feynman-variance method, named after Richard P. Feynman who suggested the theoretical basis during the early days of Los Alamos, is based on integrating the output $(*)$ of a neutron detector, placed inside or near a nuclear reactor, for a time interval $\Delta t$.

This sampling of the neutron population, under steady state operating conditions, must be repeated a large number of times, in order to obtain a suitable ensemble $\{n(\Delta t)\}$ of data to be used for the calculation of the moments of the neutron distribution. In the present procedure the computation of only the first and second order moments is necessary.

A particularly significant parameter of this distribution is the relative variance, defined as the ratio of the absolute variance (i.e. the square of the standard deviation) to the mean value. This quantity is unity for a purely Poisson process; in nuclear reactor experiments it is greater than unity because of the correlation existing among neutrons belonging to the same decay chain.

(*) This terminology is used instead of "counting a neutron detector" for sake of generality. In fact, the extension of all the pulse counter methods treated in Section 2 to the corresponding current chamber methods is straightforward, since it only implies a proportionality factor in the counting rates. This factor is essentially due to the electrical charge transferred per neutron detected. 
Thus, the excess term of the relative variance of counts depends on the reactor kinetics and the detection equipment characteristics as well as on the time interval $\Delta t$ employed for sampling the neutron population. An experimental observation of the dependence of the variance on $\Delta t$ leads to the determination of the required reactor parameters.

If $\mathrm{n}$ is the value obtained from counting a neutron detector placed inside or near a reactor, we have that

$$
V(\Delta t)=\frac{\left\langle n^{2}>-\langle n\rangle^{2}\right.}{<n>}=1+Y_{1}\left(1-\frac{1-e^{-\alpha \Delta t}}{\alpha \Delta t}\right)=1+Y
$$

where the symbols denote

$\langle n\rangle,\left\langle n^{2}\right\rangle$ first and second order moments of the distribution of samples

$\Delta t \quad$ sampling time interval

$\mathrm{Y}_{1}=\frac{\varepsilon<v(v-1)>k_{p}^{2}}{\langle v\rangle\left(1-k_{p}\right)^{2}}$

$\varepsilon \quad$ detector efficiency (counts/fission)

$v \quad$ number of neutrons emitted per fission

Equation (4) is valid in the prompt neutron kinetics approximation; other terms must be added if delayed neutron contributions have to be taken into account, which are formally analogous to the quantity $Y$. 
The technique can be profitably applied to thermal systems but suffers from prohibitive limitations in all the cases involving low detection efficiencies, $\varepsilon<\left(1-k_{p}\right)^{2}$, and therefore is practically useless for fast reactor experiments. Anyway it offers a quite valid alternative to the conventional Rossi-alpha technique, if applied to thermal reactors, since it can be rather fast and precise when the proper conditions of realization of the method are adopted. Actually, the data collection does not need to be realized in terms of the time sequence $\left.\ln _{j}(\Delta t)\right\}$. Thus, either the ensemble of data is transformed into the sample distribution $\left\{N_{k}\right\}$ where $N_{k}$ is the number of samples with content equal to $k(=0,1,2, \ldots)$ pulses and $\mathrm{N}$ is the total number of samples or data can be directly collected in distributive form.

The dependence of $Y$ on $\alpha$ is sensitive in the region $\alpha \Delta t \leqslant 1$ then tends to vanish for increasing $\Delta t$; if one want to measure $\alpha$, a limitation in the time interval is suitable that reduces the measurement time but silil allows a determination of $Y_{1}$ without any asymptotic prompt variance measurement.

The precision on $\mathrm{Y}$ depends very weakly on $\langle\mathrm{n}\rangle$ but rather strongly on $\mathrm{N}$ : this suggests that a large number of short samplings leads to a better precision than a small number of long samplings. 
The various measuring apparati may include:

1) Gated-Scaler method, in which the output pulses are counted on a time basis $\Delta t$ and stored in time sequence from $\left\{n_{j}(\Delta t)\right\}$. The operation is repeated for several values of $\Delta t$.

2) Distributive method, in which the output pulses are recorded and collected directly in the $\left\{\mathrm{N}_{k}\right\}$ form. The instrumentation needed can consist of a particular adaptation of a multichannel time analyzer.

In fact, pulses coming from the detector are fed into the address register (channel commutation switch) which is made to proceed by one step for each arriving pulse. The end of the sampling interval $\Delta t$, marked by an external timer, is fed into the data register, in order to add a count to the channel swept at that moment, and resets the address register. This way the distribution $\mathrm{N}_{k}$ vs. $\mathrm{k}$ is directly obtained along the two axes of the time analyzer.

The use of a tape recorder as intermediate stage between the detector and the data collection devices 1) and 2) can be suggested.

It allows one and the same sequence of information from the reactor to be analyzed in terms of all the differently chosen intervals $\Delta t$.

It may be interesting to note that the Feynman variance method is also suitable for plutonium experiments. The procedure is applicable because the spontaneous fission high level can be partially compensated by a reduced detection efficiency and a still sufficiently large value of $\mathrm{Y}$ due to the much smaller $\beta$ of the plutonium fuel. 


\section{THE EXPECTED NUMBER OF CORRELATED NEUTRON PAIRS METHOD}

An interesting alternative $(9,10)$ to the variance experiment is based on the determination of the ratio of the expected number of correlated neutron pairs to the expected number of random neutron pairs in a glven time interval. This parameter is strictly related to the instantaneous and mean counting rate and comes out to be quite close to the correlated relative variance. It can also profitably replace it in some experimental circumstances where the variance approach can result unfruitful, i.e. for subcritical measurements. The procedure is unsuitable for non-zero power and fast systems.

In order to understand the advantages of the method, some considerations are worthwhile about the theoretical formulation of the prompt variance analysis described by Eq. (4)

As previously emphasized, the main role is played by the quantity $Y$ which, however, suffers from the following shortcomings:

1) it is not a real reactor parameter since its magnitude depends on detector sensitivity and location inside the reactor,i.e. flux profile;

2) its value 1 s small in the time region where the maximum of correlated neutron pairs is expected, $i . e$. its value is small in the range of time intervals $\Delta t<<1 / \alpha$ where its dependence on the prompt neutron decay eigenvalue is strong and, conversely, become large where the dependence is no longer sensitive;

3) Its value is small if small detector efficiencies are available with respect to the overall fission rate, i.e. instrumentation far away from flux peak and/or small sensitivity; 
4) it has such a dependence on reactivity that it decreases with respect to that more rapidiy than $(1-\$)^{-1}$, i.e.

$$
Y(\Delta t) \vee \frac{\varepsilon D}{2 B Q} \frac{\Delta t}{(1-\$)} \quad \text { for } \alpha \Delta t<<1
$$

since increasing values of $\alpha$ demand shorter intervals to be accurately determined. Thus the Feynman-variance method cannot be applied to far subcritical systems, a smail $Y$ being prohibitive for any analysis;

5) in some assemblies such as natural uranium and light water lattices even the asymptotic and maximum value $Y_{1}$ is of the order of 0.01 and then practically useless for any significant interpretation.

A new parameter is suggested through the following formula

$$
\frac{\left(\begin{array}{l}
n \\
2
\end{array}\right)-\frac{n^{2}}{2}}{\frac{<n>}{2}}=\frac{D k_{p}^{2}}{F\left(1-k_{p}\right)^{2}}\left[\frac{\alpha \Delta t-1+e^{-\alpha \Delta t}}{\alpha \Delta t}\right]=Z(\Delta t)
$$

where $\left(\begin{array}{l}n \\ 2\end{array}\right)_{2}$ is the expected total number of neutron pairs $\frac{n}{2}>2$ is the expected number of random neutron pairs $\mathrm{n}$ is the number of counts in the time interval $\Delta t$

and

$$
Z(\Delta t)<n \equiv Y(\Delta t)
$$

In Eq. (5) one can notice that the term $\mathrm{Z}$ is

1) equal to the ratio of the expected number of correlated neutron pairs, $<\begin{aligned} & \mathrm{n} \\ & 2\end{aligned}$ - being the expected number of all the possible pairs.

2) its value vanishes for a Poisson process, as well as the term $Y$ does, according to the absence of any correlated pair in that circumstance. This is also equivalent to the fact that $Z$ vanishes if no multiplication exists.

3) in direct opposition to $Y, Z$ is large in the range of time intervals $\Delta t<<1 / \alpha$ and has a strong dependence on the value of $\alpha$, as can be seen from the following formula, obtained from Eq. (5) for $\alpha \Delta t<1$ 


$$
z(\Delta t) \sim z(0) \quad 1-\frac{a \Delta t}{3}
$$

4) for very short time intervals the term $Z$ does not vanish, opposite to $Y$, since no correlation is detectable for vanishing sampling intervals. Conversely $Z$ remains finite and tends to a maximum since the chances of correlated pairs increase with respect to these of random pairs. The limit value $\mathrm{Z}(0)$ coincides with the B/A of the Rossi-alpha experiment (see $\mathrm{Eq} .1$ ) and therefore offers significant theoretical basis and the possibility of a comparison between the two techniques.

5) for very long time intervals, but still in the prompt neutron approximation, the term $Z$ tends to vanish, since the number of correlated pairs saturates and practically all random pairs are detected.

6) the term $Z$ is a characteristic physical parameter of the reactor, since it does not depend on detector location and sensitivity but only on reactivity and power (source) level as well as on the sampling interval $\Delta t$.

7) The dependence of $Z$ on reactivity is also given by Eq. (5) and appears to be a direct function of $\alpha$, i.e. the slope of the behaviour, since the initial value $z(0)$ is approximately constant for decreasing reactivities. In fact, it can be written as

$$
Z(0) \sim \frac{D<\nu>}{2 S l}
$$

where $S$ is the effective source strength due to spontaneous fissions, plus any other additional source.

\section{THE BENNETT VARIANCE METHOD}

The Bennett-variance method, $(11,12)$ named after Edgar F. Bennett who suggested and performed the experiment in 1960 , is not simply another alternative to the conventional variance technique since it exhibits further advantages and possibilities with respect to the previous one. 
The procedure is based on the calculation of the mean square value of the difference of integrated flux over two consecutive equal time intervals. The quantity obtained contains as much information about the reactor parameters as the variance but does not feature the mathematical property of divergence at criticality, which is characteristic of the quantity $\mathrm{Y}$.

If the definition of $n_{j}(t+j \Delta t)$ is generalized to the meaning of integral of reactor noise over the interval $t+j \Delta t-t+(j+1) \Delta t$ in order to include also a current chamber experiment, we have that

$$
\frac{\left\langle\left(n_{j}-n_{j-1}\right)^{2}\right\rangle}{2<n>}=1+Y_{1}\left(1-\frac{\frac{3}{2}+\frac{1}{2} e^{-2 \alpha \Delta t}-2 e^{-\alpha \Delta t}}{\alpha \Delta t}\right)=1+W
$$

which is valid in the usual prompt neutron approximation and has some close similarities to the Feynman vartance formulation.

The technique can be applied to thermal reactors but not to fast reactors because of the 1 imitations due to the required high detection efficiency as in the conventional variance experiment.

Two different measuring apparati must be considered according to the type of experiment involved. They actually differ in the whole structure, although the final output can be 1dentical; one is based on a pulse counter and is likely to be applled to zero-power experiments, the other including the use of a current chamber can also be applied to non-zero power measurements. They are 
1) Pulse counter method: The entire procedure can be the same as in the Feynman variance experiment, only with the difference of an output necessarily in time sequence form, since the distribution $\left\{\mathrm{n}_{j}\right\}$ of the collected data is now a foundamental information to be used, if one wants to minimize the data collection time. The alternative version regarding the use of a tape recorder may or may not be taken into account without or with the already discussed inconveniences. The distributive method cannot be applied because it cancels the information chronology, unless a more sophisticated data collection is adopted, e.g., the distribution of the difference between consecutive samples.

2) Current chamber method: The basic instrument is a gamma-compensated current chamber of the type generally adopted as flux monitor in nuclear reactor control. The chamber output is fed into a DC amplifier and thereafter into a double RC integrating circuit. The two parts of this block work alternatively in order to make possible an integration of reactor noise in 'actually' successive intervals, viz. without any $\mathrm{lag}$, as theory imposes. In fact at the end of each fixed time interval, the result of the integration is displayed by discharge via a suitable recorder. An output in sequence form is collected, ready to be treated according to the left hand side of $\mathrm{Eq} \cdot(6)$.

THE THIRD ORDER ANALYSIS METHOD

Further and recent investigations on the neutron fluctuation phenomena have considered the possibility of reaching additional information via a third order moment analysis of the distribution of counts as an extension of the second order procedures previously discussed. For instance, the mathematical formulation shows $(13,14)$ that, from a strictly theoretical point of view, experiments of this type yield $\beta$ and $\Lambda$ separately without any need of static measurements. And this would really be a novelty in reactor parameter measurements. 
BNWL -875

Several types of analysis have been proposed according to the several combinations of third order moments which can be adopted in manipulating the collected data.

We shall report here two of the most interesting formulae. If $\mathrm{n}$ is the number of counts from a neutron detector in a sampling time interval $\Delta t$, we have that

$\frac{\left\langle n(n-1)(n-2)>-3<n>\left\langle n(n-1)>+2<n>^{3}\right.\right.}{3<n>}=Y_{1}^{2}\left(1+e^{-\alpha \Delta t}-2 \frac{1-e^{-\alpha \Delta t}}{\alpha \Delta t}\right)$

and

$\frac{\left\langle n_{i} n_{i+j}\left(n_{i}-n_{i+j}\right)\right\rangle}{\langle n>}=\frac{\varepsilon^{2}\langle\nu(\nu-1)(\nu-2)\rangle}{3<\nu\rangle\left(1-k_{p}\right)^{3}}(\alpha \Delta t)^{2} e^{-\alpha j \Delta t}\left(1-e^{-\alpha j \Delta t}\right)$

Equation (7a) and (7b) are valid in the prompt neutron approximation and are condensed versions of more complicated theoretical formulations including additional terms. However these have neglegible numerical amount and are likely to be unobservable in the related experiments.

Equation ( $7 b$ ) is valid under the condition $\alpha \beta>>1$, which is satified only for fast reactor experiments.

Knowing $\mathrm{Y}_{1}$ and the constant coefficient of Eq. (7b) allows a direct determination of $\beta$ to be realized and, therefore, an independent measurement of $\Lambda$ from the ratio $\beta / \alpha$ at criticality, to be obtained. The stringent necessity of a very high efficiency and the difficult realization of this task in fast reactor experiments are the contrasting factors at the basis of such experiments, which potentially can yield extremely interesting 
BNWL -875

26

information.

The only experiment performed with those purposes has shown that, for the moment, the horizon of these procedures is not larger than that offered by the second order methods and that a separate determination of $\beta$ and $\Lambda$ is based on the detection of events (a triplet of neutrons originated from the same fission event) which is too rare and unfrequent to make it possible, at least in thermal reactors.

Thus, at the present time, the third order experiment is just another type of analysis which can be done on the same set of data useful for the second order experiments and in some cases-e.g. those in which a very high efficiency is available - can allow a more adequate precision to be reached in comparison with the various variance procedures.

THE COVARIANCE METHOD

The covariance method $(15,16)$ is based on simultaneously counting two neutron detectors, placed inside or near a nuclear reactor, for a time interval $\Delta t$.

This sampling of the neutron population must be repeated a large number of times in order to obtain the ensemble $\left\{n_{1}(\Delta t), n_{2}(\Delta t)\right\}$ of data to use for the calculation of the covariance of the two counting variables $n_{1}(\Delta t)$ and $\mathrm{n}_{2}(\Delta t)$ from detector 1 and 2 , respectively.

The covariance is defined as the difference between the average product of the variables and the product of the mean values of them. It vanishes if the two quantities are uncorrelated: otherwise it is a good index of the degree of coherence or correlation between them. 
If we define the covariance $\operatorname{Cov}(\Delta t)$ as previously said

$$
\operatorname{Cov}(\Delta t)=\left\langle\left(n_{1}-\left\langle n_{1}\right\rangle\right)\left(n_{2}-\left\langle n_{2}\right\rangle\right)\right\rangle=\left\langle n_{1} n_{2}\right\rangle-\left\langle n_{1}\right\rangle\left\langle n_{2}\right\rangle
$$

we have that

$$
\frac{\operatorname{Cov}(\Delta t)}{<n_{1}>}=\frac{\varepsilon_{2} D k_{p}^{2}}{\left(1-k_{p}\right)^{2}}\left(1-\frac{1-e^{-\alpha \Delta t}}{\alpha \Delta t}\right)
$$

or, alternatively,

$$
\left.\frac{\operatorname{Cov}(\Delta t)}{<n_{2}>}=\frac{\varepsilon_{1} D k_{p}^{2}}{\left(1-k_{p}\right)} 2^{2}-\frac{1-e^{-\alpha \Delta t}}{\alpha \Delta t}\right)
$$

where the symbols $\varepsilon_{1}$ and $\varepsilon_{2}$ denote the detector efficiencies.

When compared with the classical Feynman-variance formulation

[see Eq. (4)] concerning the single detector experiment, Eq. (8a)

and ( $8 b$ ) show the absence of the Poissonian relative variance, which

contributes an additional term equal to unity to the quoted formula of the autovariance - or, simply, variance - of counts.

As expected, the adoption of two detectors eliminates this

uncorrelated term, gives only the required coherent amount of information and so enables the experiment to be profitably performed in low detection efficiency conditions, e.g. detector in peripheral reactor zone, in-core inscrumentation in fast systems.

The experiment emphasizes some advantages not obtainable with the use of a single detector and the computation of the variance. In fact, the adoption of two counters relieves the stringent necessity of a high detection efficiency, unlike the major part of all the variance-type 
experiments. Actually, in order to minimize the errors due to the finite number of samples, the detection efficiency cannot be too low.

As far as we know, no measurements of this type have been realized or, at least, no published matter on the subject is available.

The mathematical formulation of the method immediately suggests that the practical realization of the covariance experiment is based on doubling the measuring apparatus of the classical Feynman experiment.

In fact, two lines of the type named 'gated scaler method' in that section are sufficient to realize the procedure. A necessary condition is that a common time marker triggers both the scalers so that the successive time intervals $\Delta t$ are opened simultaneously for the two counting operations. The distributive method cannot be applied here since the two distributions $\left\{n_{1}(\Delta t)\right\}$ and $\left\{n_{2}(\Delta t)\right\}$ are not required and only the joint distribution $\left\{n_{1}(\Delta t), n_{2}(\Delta t)\right\}$ is needed for the off-line data computation. The latter distribution could be directly recorded if a two parameter analyzer could be used.

Section 3 : Differentiae Analysis of Counting Distirbutions THE ZERO COUNT PROBABILITY METHOD

The zero probability technique $(17,18)$ is based on the determination of the relative variance of counts from a neutron detector by measuring the fraction of 'empty' samples for different values of the interval $\Delta t$ over the total number of samples taken during the experiment. 
BNWL-875

If we call $\mathrm{p}_{\mathrm{o}}(\Delta \mathrm{t})$ the probability of no counts in a time interval $\Delta t$, we have that

$$
\mathrm{p}_{\mathrm{o}}(\Delta \mathrm{t})=\exp \left\{-\frac{\mathrm{A}}{2} \overline{\mathrm{B}}\left[(\gamma-1) \alpha \Delta t+2 \frac{(\gamma+1)^{2}-(\gamma-1)^{2} \mathrm{e}^{-\alpha \gamma \Delta t}}{4 \gamma}\right]\right\}
$$

where $A$ and $B$ have been given in $\mathrm{Eq} .(1)$ and the symbol $Y$ denotes

$$
\gamma=\left(1+2 Y_{1}\right)^{1 / 2}
$$

and is immediately related to Eq. (4).

Measurements need to be performed at extremely low counting rates so that the probability of no counts can be significantly different from zero and are most likely to be applicable to thermal systems where adequate detection efficiencies are available.

The procedure is rather straightforward, unlike some other variance methods, and needs very little data reduction since the essential data are directly given by the measuring apparatus.

Measuring apparati of different type can be used for such an experiment. Possibly the most straightforward procedure is based on an anticoincidence circuit. It operates as follows: the pulses from the discriminator are fed into the anticoincidence which gives an output only if, during the interval marked by an external timer, no neutron pulse arrives. The pulse at the finish of the interval resets the anticoincidence and prepares it for the same operation in the successive interval.

At the end of the procedire the number of output pulses gives the value $N_{0}$ of samples with no pulse content. The total number $N$ of samples is given by the ratio of the measurement time $T$ to the time interval $\Delta t$. 
BNWL-875

30

Once the relative variance of counts has been obtained the procedure features the same characteristics and implications as the methods described in Section 2.

THE POLYA MODEL METHOD

The Polya model method is based on the determination of the relative variance of counts from a neutron detector by directly comparing the frequency distribution of counts, $i . e$. the ensemble of the fractions $f_{k}$ of samples with content equal to $k=0,1,2, \ldots$ pulses over the total number of samples, with the theoretical distribution function.

This has been shown to be well approximated by the probability profile of the Polya urn model, which has been introduced by G. Polya to describe some aspects of the theory of games and the aftereffects of contagious deseases .

If we call $\mathrm{p}_{\mathrm{k}}(\Delta \tau)$ the probability of couniing $k$ pulses in a time interval $\Delta t$, we have that

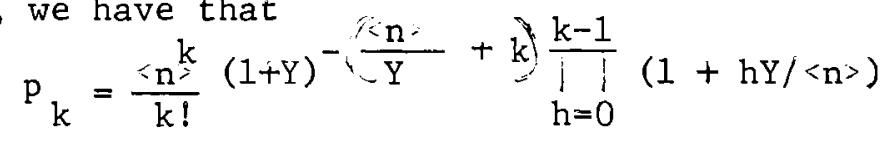

or, in a simpler recursion formula,

$$
p_{k}=\frac{n=+Y(k-1)}{k(1+Y)} p_{k-1} \text { and } p_{o}=(1+Y)^{-<n>/ Y}
$$

where the dependence on $\Delta t$, present in $n$ and $Y$, has been dropped out for simplicity.

The method is obviously still efficiency sensitive and can be applied only to thermal systems where the relative variance of counts is significant1y greater than unity even in the prompt neutron time domain. 
Equipment needed for other variance procedures is evidently useful even for this experiment. In particular, the distributive methods is we11 suited to give directly the frequency distribution of counts in terms of the quantities $N_{k}(\Delta t)$ which denote the number of pulses in the $(k+1)$-th channel of the multiscaler, i.e. the number of the time interval with content equal to $k$ pulses.

The 'gated scaler method' is not suitable here since it would need too much data reduction in order to transform the raw output from the time sequence form into the distributive form.

The procedure features some advantages with respect to other related statistical techniques, viz. the Feynman variance method and the zero probability method, since it avoids part of the uncertainties which might affect them.

In fact, the first one calculates $\mathrm{Y}$ as a difference between the total relative variance and the random component of the relative variance which is equal to unity. This way noise components not related to the neutron population fluctuation phenomena might be included and there is no way to eliminate them unless the actual distribution of neutrons is known.

The second method is too restrictive, since only one point, viz. $\mathrm{N}_{0}$ of the experimental distribution is employed to derive the variance of counts and is therefore atfected by all the possible consequences of a zero-dimensional measurement. 
OTHER RELATED PROBABILITY METHODS

A certain number of procedures intermediate between the zero-count probability method and the Polya model method are commonly used. They are based on simple and suggestive properties of the adopted probability distribution function and can be summarized as follows. Zero-count to one-count probability ratio method $(21,22)$

If the recursion formula of $\mathrm{Eq}$. (10) is recalled and limited to $\mathrm{k}=1$ one finds that

$$
\frac{<\mathrm{n}=}{\mathrm{p}_{1}}=1+\mathrm{Y}
$$

which suggests a useful method to determine the relative variance of counts.

The Furuhashi-Izumi probability method $(, 10)$

If the ratio of $p_{0}$ to $p_{1}$ is suitable to yield the quantity $1+Y$, typical of the Feynman-variance experiment, a particular combination of $\mathrm{p}_{\mathrm{o}}, \mathrm{p}_{1}$ and $\mathrm{p}_{2}$ leads to the determination of the index $1+z$ introduced by Furuhashi and Izumi in their data treatment of the Feynman variance experiment [see Section 2]

In fact, recalling Eq. (10), one can promptly obtain

$$
\frac{2 \mathrm{p}_{0} \mathrm{p}_{2}}{\mathrm{p}_{1}^{2}}=1+\mathrm{Z}
$$

which establishes a measuring procedure of the parameter $Z$ for different time intervals $\Delta t$. From the $Z$ vs. $\Delta t$ plot the quantities $\alpha$ and $B / A$ can 
be obtained through the usual least square fit methods.

Both the discussed techniques feature a fundamental limitation since they need more complicated equipment than that used for the zerocount probability experiment (usual variance equipment instead of the anticoincidence circuit) and, simultaneously, employ only few points of the observed frequency distribution $\left\{\mathrm{N}_{k}\right\}$ in order to reach a significant value of $Y$ or $Z$. Obviously the determination of the complete frequency profile $\left\{\mathrm{N}_{k}\right\}$ needs the same time and the same apparatus and certainly gives a more accurate value of $\mathrm{Y}$ and/or $\mathrm{Z}$.

Even-and/or odd-count probability method $(23,24)$

The previously mentioned inconvenience is not exhibited by another procedure which can be realized with simple equipment and still gives useful information about the relative variance of counts in a certain interval $\Delta t$. It is based on probability of counting an even and/or an odd number of neutron pulses in a given time interval $\Delta t$.

If the relationship between the probability $p_{k}$ and its generating function $G(s)$ is recalled, we can derive that

$$
\mathrm{P}_{\text {even }}{ }^{-\mathrm{p}_{\text {odd }}}=\mathrm{G}(-1)
$$

when $p_{\text {even }}\left(p_{\text {odd }}\right.$ ) is the probability of an even (odd) number of pulses in the time interval $t$.

If Eq. (11) is applied to various generating functions, we have that 


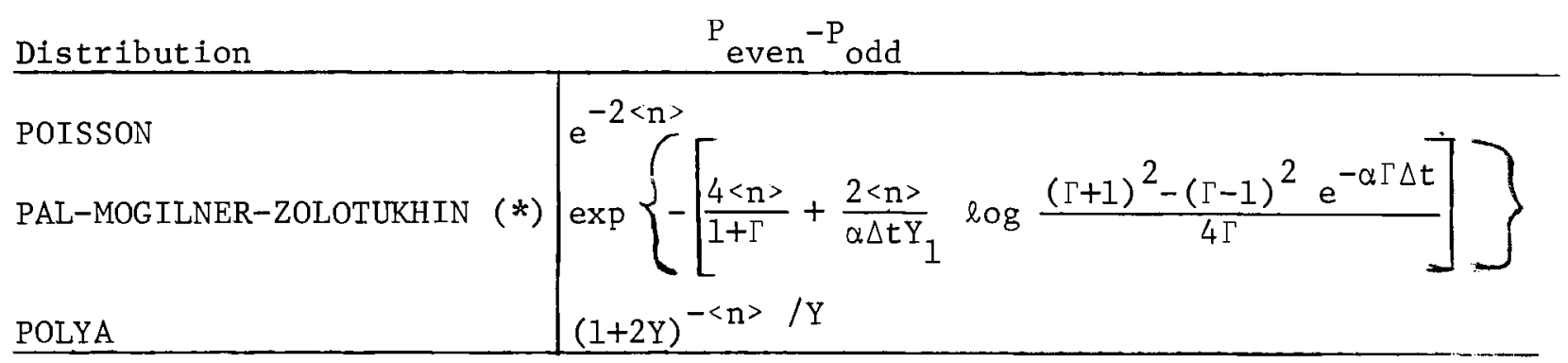

where

$$
\Gamma=\left(1+4 Y_{1}\right)^{1 / 2}
$$

The described equations suggest a method based on the measurement of the quantities $\mathrm{N}_{\text {even }}$ (i.e,, the number of samples with content equal to an even number of pulses), or $\mathrm{N}_{\text {odd }}$, and $\langle\mathrm{n}\rangle$, in order to determine $\mathrm{Y}$.

The advantage of the technique consists in the data collection, which is extremely strightforward and ignores the numerical content of each analyzed counting interval $t$, being this way realizable by a one-bit counter. In fact, the measuring apparatus can consist of a flip-flop circuit into which pulses from the neutron detector are fed. The flip-flop is enabled and reset by an external timer and its output may be read just in terms of $\mathrm{N}_{\text {even }}$ (or $\mathrm{N}_{\text {odd }}$ ) since the simple relations hold

$$
\mathrm{N}_{\text {even }}{ }^{-\mathrm{N}} \text { odd }=2 \mathrm{~N}_{\text {even }}-\mathrm{N}=\mathrm{N}-2 \mathrm{~N}_{\text {odd }}
$$

(*) The Pal-Mogilner-Zolotukhin distribution is very close to the actual distirbution of neutron counts from a detector placed inside a nuclear reactor. However, it is explicitly known only under the form of its probability distribution generating function and not yet in terms of the probabilities $p_{k}$. This is the reason why the Polya distribution is used to replace it. 
The even-and odd-count probability method has introduced a new approach for the satistical techniques, which is based on the possibility of measuring the same parameters as the previous precedures, with an approximately comparable precision, although the information collected is limited to a minimum (only one bit per sample).

That is to say that, for a Poisson distribution, the mean value can be determined even if only the parity of the events (even or odd number of counts in a given time interval) is recorded and any other numerical information is disregarded.

In the case of a Pal-Mogilner-Zolotukhin (or Polya) distribution, the relative variance of counts can be obtained by simply recording the parity of events (independently from their time sequence) and the longtime averaged mean value, which is easy to be separately measured by a gated scaler operating during the whole measurement time.

This new approach finds other interesting applications in the polarity techniques, based on the collection of only the sign of the fluctuations of a steady state operating reactor power level with respect to their mean value.

Even in these cases, some fundamental reactor physics quantities, as the auto-and cross-correlation or the covariance of counts can be obtained as well, although the numerical analysis of the neutron population phenomena is completely bypassed and only one bit of information per sample (i.e., the polarity, the sign of the difference between the instant level and the average level of reactor flux) is recorded.

These methods are extensively treated in Section 4. 
BNTL-875

\section{TIME INTERVAL FROM A RANDOM ORIGIN METHOD}

The method $(25,26)$ is based on the determination of the time interval distribution between a random ly chosen instant in the reactor life and the first arriving pulse from a neutron detector.

The basic difference in comparison with the Rossi-alpha or auto-correlation experiment lies essentially in when the time analysis is made to start, $i$,e in how the triggering pulse is chosen.

The former experiment needed a neutron pulse to make the analyzer start the sweep, the latter one can be triggered at random, $i_{n} e$, the sequence of triggering pulses obeys a Poissonian lawn

This experiment differs also from the zero-probability method where the involved analysis concerns the probability of no count in a given time interval (and therefore, the probability of a count any time later) while the method discussed here implies the additional condition that one count occurs at the end of the given interval.

If we indicate with $\pi(t) d t$ the probability that after a time $t=0$ chosen at random, the first pulse arrives between $t$ and $t+d t$, we have that

$$
\pi(t) d t=2 A p_{0}(t) \frac{\gamma+1+\left(\gamma-1 ; e^{-\alpha \gamma t} d t\right.}{(\gamma+1)^{2}-(\gamma-1)^{2} e^{-\alpha \gamma t}}
$$

where $p_{0}(t)$ is the probability of no counts in the time interval $0 \rightarrow t, i=$ the probability that after a time $t=0$ chosen at random, the first pulse arrives at any time greater than $t$. 
The method is efficiency limited-and therefore cannot be applied to fast reactors where the relative variance of counts from a neutron detector is significantly different from unity (the variance of a random process) only for extremely high (usually unavailable) detection efficiency.

The measuring apparatus must essentially work as a clock of the time interval between a random origin and the first arriving pulse.

A time-analyzer clearly fits the purpose: it must be triggered at random. The pulses emitted by a long half-life radioactive substance may be one of the ways. Actually the time-analyzer sweep does not start with that external pulse but with the first arriving pulse from the interval oscillator after that pulse.

Since there is no correlation between the triggering pulse and the sweep-start pulse, the introduced delay between the two pulses does not affect the correct realization of the procedure.

When the first pulse arrives from the neutron detector the analyzer adds a count in the channel swept at that moment and resets the address register to make it ready for a new cycle. It begins with the first arriving pulse, from the random sequence generator after the end of the described operation.

Thus only one event per sweep is recorded and the time distance of a pulse from the origin of the time axis (zero channel) is actually the time interval from a random origin. The data collection is repeated for a large number of cycles and accumulated in the time-analyzer. 
The procedure is effective in thermal reactor experiments, where the Rossi-alpha method must usually be discarded, and features a faster and more direct realization than the zero-count probability method, since only one run is needed to describe the whole $\pi(t)$ vs. $t$ behaviour unlike the former method.

PULSE TO PULSE TIME INTERVAL METHOD

The method $(27,28)$ is based on the determination of the time interval distribution between consecutive pulse or, more rigorously, of the probability that after a pulse at $t=0$ the successive pulse comes within $t$ and $t+d t$.

The basic difference in comparison with the Rossi-alpha experiment lies in the analyzed distribution which is that of counts following a certain triggering pulse (a neutron detection) in that case and that of the immediately successive pulse after the trigger in this case,

Thus the second probability differs from the first one because it contains the additional condition that no pulse occurs in the time interval $0 \rightarrow t$. It is expected that for very low detector efficiency and counting rates the two experiments tend to coincide.

If we denote with $p(t) d t$ the probability that after a pulse at $t=0$ the next pulse comes within $t$ and $t+d t$, we have that

$$
p(t) d t=C_{1}(t) d t+C_{2}(t) e^{-\alpha \gamma t} d t
$$


where the quantities $C_{1}(t)$ and $C_{2}(t)$ are given by

$$
\begin{aligned}
& C_{1}(t)=4 A P_{0}(t)\left[\frac{\gamma+1+(\gamma-1) e^{-\alpha \gamma t}}{(\gamma+1)^{2}-(\gamma-1)^{2} e^{-\alpha \gamma t}}\right]^{2} \\
& C_{2}(t)=\frac{8 A P_{o}(t) \gamma^{2}}{\sigma(\gamma+1)^{2}-(\gamma-1)^{2} e^{-\alpha y t 2}}
\end{aligned}
$$

and the new symbols can be expressed in terms of the previously introduced nomenclature as

$$
\sigma=\frac{\mathrm{S} \Lambda}{\langle\nu\rangle \mathrm{D}}=\frac{\mathrm{A}}{2 \mathrm{~B}}[\text { see Eq. (1)] }
$$

From a theoretical viewpoint the method discussed here can be regarded as effective as the Rossi-alpha precedure in the case of fast reactor experiments but greatly to be preferred for thermal experiments since the previous one is rather time consuming and the autocorrelation method needs elaborate off-line computation.

Another advantage of the technique is that it does not suffer extremely limiting inconvenience if only low detection efficiency and/or high power (source) level are involved, unlike the major part of other statistical methods. In fact, even in these extreme cases the final formula still contains significant information about the required reactor parameters.

The measuring apparatus must essentially work as a clock of the time interval between consecutive pulses.

The previously described stop-watch time analyzer can be used but half of the data is lost, as it has been already explained (see Part I). For a 
full utilization of the arriving information an ad hoc adaptation of a time analyzer must be realized which can work as follows:

The sequence of counts coming out from the discriminator is fed into the time analyzer so that the first arriving pulse triggers the sweep of the channels. Particular attention must be dedicated to the correction of the delay effect introduced by the completely random relationship between the arrival of the triggering pulse and the first pulse from the analyzer internal timer, which actually makes the sweep start.

When a second pulse occurs, the analyzer adds a count to the channel activated at that instant, resets the address register and triggers another sweep. This three-part operation subtracts time, in all the three phases, to the effective interval between successive pulses and yields delay effects which have to be taken into account in order to restore the true time correlation among the pulses.

Thus, only one event per sweep is recorded and the suitably corrected time distance of a pulse from the origin of the time axis (zero channel) is actually the separation between that pulse and the preceding one. The process is repeated a large number of times and accumulated in the time analyzer.

No correction formula is available at the moment for the various delay effects. 
Section 4: Correlation of the Signs of Fluctuations about a Mean Value THE POLARITY AUTO-and CROSS-CORRELATION METHOD

The polarity correlation method $(29,30)$ is based on the determination of the autocorrelation (cross correlation) function of one (two) neutron detector outputs, where this output is not numerical but simply a +1 or a -1 according as the detector fluctuations are above or below their mean value, at that instant.

The method is particularly useful to apply to non-zero power systems where pulse counters cannot be adopted because of their limited resolution time. Furthermore the cross correlation technique ameliorates the inconvenience of low detection efficiencies in fast reactors, thus extending the procedure to all types of nuclear systems.

If $x_{1}(t)$ and $x_{2}(t)$ are the counting rates from two neutron detectors inside or near the reactor, the numerical cross correlation function and the polarity cross correlation function are defined as

$$
\begin{aligned}
& C(\tau)=\left\langle x_{1}(t) x_{2}(t+\tau)\right\rangle \\
& P(\tau)=\left\langle\operatorname{sgn} x_{1}(t) \operatorname{sgn} x_{2}(t+\tau)\right\rangle
\end{aligned}
$$

respectively. The mean values $\left\langle x_{1}(t)\right\rangle$ and $\left\langle x_{2}(t)\right\rangle$ are assumed to vanish and the new symbols denote

$$
\operatorname{sgn} x(t)=\begin{aligned}
& +1 \text { if } x(t)>0 \\
& -1 \text { if } x(t)<0
\end{aligned}
$$

The dependence of $C(\tau)$ on reactor parameters is given in Eq. (3). 
If the amplitude distribution of $x_{1}(t)$ and $x_{2}(t)$ is assumed to be Gaussian, the two functions are related by

$$
\sin \frac{\pi}{2} P(\tau)=\frac{C(\tau)}{C(0)}=R(\tau)
$$

The method needs a polarity monitor (a detector of the sign of the difference between the fluctuations and their mean value) and the usual data reduction, typical of the correlation algorithm, essentially based on the operations of delay, multiplication and addition. It is easy to understand that they are greatly simplified if the involved numbers are only positive and negative unity.

Although the polarity technique has been essentially introduced in order to simplify the process of quantization necessary when continuous time signal must be digitized, the procedure is likely to be also applied to output signals which are already in digital form, e.g., number of pulses. Generally the procedure can be of the following type: the current from an ion chamber, placed inside or near the reactor, is sampled at a certain rate. The output of the integrating circuit is compensated by the long time averaged mean value (obtained, for instance, through a long time-constant counting-rate meter) and gives $a+1$ or $a-1$ according to the positive or negative sign of the outcoming signal.

If the on-line compensation is not possible, the quantization to a polarity signal can be realized off-line by a computer, once the various samples have been suitably digitized. 
An improved procedure has been recentiy adopted which is based on an instant sampiing of the reactor fiuctuations. In fact, the noise is separaied from the DC component, amplified and then fed into a Schmitt trigger adjusted to switch at zero volts. The trigger output is sampled every desired period

\section{THE COVARIANCE METHOD VIA POLARITY DETECTION}

The covariance experiment ${ }^{(16)}$ has been already introduced in section 2 as a two-detector procedure. Its application can benefit from further improvement if the meaning and the role of the covariance of a joint distribution of two variables is recalled and appropriately used in order to optimize the data collection process.

Assume that a two-detector output is integrated over a time interval $\Delta t$ and that the joint distribution of these outputs is $\left\{x_{1}(\Delta t)_{2} x_{2}(\Delta t)\right\}$

The present method is based on the statement that no numerical information about the two variabies is indispensable for the calculation of the covariance, since only knowing the sign of the two variables with respect of their mean values and using some properties of the probability distribution function aliow a simpler and faster determination of the covariance.

Let us indicate with $\operatorname{sgn}\left[\mathrm{x}_{1}(\Delta t)-\mathrm{x}_{1}(\Delta t)>\right], \operatorname{sgn}\left[\mathrm{x}_{2}(\Delta t)-\left\langle\mathrm{x}_{2}(\Delta t)>\right]\right\}$ the joint distribution of the polarity variables. Four types of pairs are clearly possible according to various combinations of signs, viz, + -- t- Denote with $\mathrm{P}_{1} \mathrm{P}_{2} \mathrm{P}_{3}$ and $\mathrm{P}_{4}$ the probability of appearence of each pair. 
If the two variables are positively (negatively) correlated, $\mathrm{P}_{1}$ and $P_{3}\left(P_{2}\right.$ and $\left.P_{4}\right)$ are greater than $P_{2}$ and $P_{4}\left(P_{1}\right.$ and $\left.P_{3}\right)$ according to the amount of coherence involved, which is clearly the covariance of the two variables for the adopted integration interval $\Delta t$.

Since experimental quantities very close to $P_{i}(i=1,2,3,4)$ can be measured in terms of the observable frequencies of the four types of pairs and the dependence of the covariance on reactor kinetic parameters has been previously described, the experiment can be interpreted as soon as one finds the dependence of the $P_{i}$ on the covariance $\operatorname{Cov}(\Delta t)$.

If $x_{1}(\Delta t)$ and $x_{2}(\Delta t)$ are the integrated outputs of two simultaneously observed detectors over a time interval $\Delta t,\left\langle x_{1}(\Delta t)\right\rangle$ and $\left\langle x_{2}(\Delta t)\right\rangle$ are their mean values over the whole ensemble of samples and two new variables are introduced,

$$
\operatorname{sgn}(i)=\operatorname{sgn}\left[x_{i}(\Delta t)-\left\langle x_{i}(\Delta t)\right\rangle\right]=\left\{\begin{array}{cc}
+1 & \text { if } x_{i}(\Delta t)>/\left\langle x_{i}(\Delta t)>\right. \\
-1 & \text { if } x_{i}(\Delta t)<\left\langle x_{i}(\Delta t)>\right.
\end{array}\right\}
$$

the joint distribution $\{\operatorname{sgn}(1), \operatorname{sgn}(2)\}$ can be considered. The pair may exhibit four possible combinations, namely $H_{,}-+_{0}--_{2}+-$, whose probabilities can be denoted as $\mathrm{P}_{1}, \mathrm{P}_{2}, \mathrm{P}_{3}$ and $\mathrm{P}_{4}$,

If the joint distribution function of $x_{1}(\Delta t)$ and $x_{2}(\Delta t)$ is assumed to be a bivariate normal distribution, then

$$
\begin{aligned}
& P_{1}=1 / 4+\frac{1}{2 \pi} \arcsin [\operatorname{cov}(\Delta t)] \\
& P_{2}=1 / 4-\frac{1}{2 \pi} \arcsin [\operatorname{cov}(\Delta t)]
\end{aligned}
$$




$$
\begin{aligned}
& \mathrm{P}_{3}=1 / 4+\frac{1}{2 \pi} \arcsin [\operatorname{cov}(\Delta t)] \\
& \mathrm{P}_{4}=1 / 4=\frac{1}{2 \pi} \arcsin [\operatorname{cov}(\Delta t)]
\end{aligned}
$$

where $\operatorname{cov}(\Delta t)$, i.e the covariance of the two variables, has been explicitly formulated in Eq. (8). That is to say

$$
\operatorname{cov}(\Delta t)=\sin \frac{\pi}{2}\left[\mathrm{P}_{1}+\mathrm{P}_{3}-\left(\mathrm{P}_{2}+\mathrm{P}_{4}\right)\right]
$$

Being a two-detector experiment, the covariance method does not (*) suffer any Iimitation due to low etficiency detection and can be appiled to fast reactor-noise analysis, unilke ail the other one-detector variance procedures.

Since the detection of only the sign of the variables is needed, a pulse counter is dispensable and cherefore the technique is expected to be applicable even tc non=zero power reactors.

For this purpose, the noise can be separated from the DC component, amplified and then integrated by one of the two alternatively working RC integrating circuits (as in the Bennett-variance experiment). The polarity, that is to say the sign of the integrated output is then recorded in sequen, e $\pm 0 \mathrm{~m}$,

An idencical procedure is realized in parallel for the other detector so that the simultaneous one-bit information, about the sign of the integrated noise with respect to the mean fluctuation level; is recorded.

A system can be studied which would be able to give directly the value of the four frequencies corresponding to the four possible pair outouts.

${ }^{(*)}$ However, because of the finite number of samples, the detection efficiency cannot be too low. 
• 
BNWL-875

\section{REFERENCES}

(1) J.D. ORNDOFF- "Prompt Neutron Periods of Metal Critical Assemblies", Nucl. Sci. Eng. 2, 450 (1957)

(2) D. BABALA - "On the Theory of Rossi-alpha Experiment in Reactor Noise Studies", Nucl. Sci. Eng. 26, 418 (1966)

(3) S. CHWASZCZEWSKI et al. - "Improved Method for Prompt-Neutron-Period Measurements", Nuc1. Sci. Eng. 25, 201 (1966)

(4) N. PACILIO - "Statistica1 and Pulsed Experiments in Organic Moderated and Reflected Assembles", Energia Nucleare 15, 129 (1968)

(5) H. BORGWALDT, D. STEGEMANN- "A Common Theory for Neutronic Noise Analysis Experiments in Nuclear Reactors:, Nukleonik $7,106(1965)$

(6) T. NOMURA et al. - "Reactivity Measurements by the Two-Detector CrossCorrelation Method", AEC Symposium Series 9,217 (1967)

(7) R.W. ALBRECHT -"The Measurement of Dynamic Nuclear Reactor Parameters using the Variance of the Number of Neutrons Detected", Nuc1. Sci. Eng. 14, 153 (1962)

(8) N. PACILIO - "Short-Time-Variance Method for Prompt-Neutron-Lifetime Measurements", Nucl. Sci. Eng. 22, 266 (1965)

(9) S. IZUMI - "A Study on the Counting Statistics of Neutrons in a Multiplying Medium", Master's Thesis, Tokyo Institute of Technology (1967)

(10) A. FURUHASHI, S. IZUMI - "A Proposal on Data Treatment in Feynmanalpha Experiments", J. Nuc1. Sci. Tech. TOKYO 4,99 (1967)

(11) E.F. BENNETT - "The Rice Formulation of Pile Noise", Nucl. Sci. Eng. $8,53(1960)$

(12) J.B. DRAGT - "Bias Due To Finite Measuring Time in Nuclear Reactor Noise Analysis", RCN 67-064, Reactor Centrum Nederland, (1967)

(13) J.B. DRAGT - "Threefold Correlations and Third Order Moments in Reactor Noise", Nukleonik 10. 7 (1967)

(14) E. TURKAN, J.B. DRAGT - "Experimental Study of Different Techniques for Analyzing Reactor Noise Measured by a Neutron Counter", RCN 67-075, Reactor Centrum Nederland (1967) 
BNWL-875

(15) C.E. COHN - remark on a paper by Christensen et al. "A Review of the NORA Project Noise Experiments", AEC Symposium Series 9, 530 (1967)

(16) N. PACILIO-"Reactor-Noise Analysis : the covariance method via polarity detection" Nucl. Sci. Eng. (in press)

(17) L.I. PAL - "Statistical Theory of Neutron Chain Reactions (part III)", Acta Phys. Hung. 14, 369 (1962)

(18) V.G. ZOLOTUKHIN, A.I. MOGILNER- "The Distribution of Neutron Counts From a Detector Placed in a Reactor", At. Energy (USSR) 15,11 (1963)

(19) V.G. ZOLOTUKHIN, A.I. MOGILNER - "Distribution of Neutron Counts Recorded by a Neutron Detector in a Reactor", at. Energy (USSR) 10,379 (1961)

(20) N. PACILI0-"The Polya Model and the Distribution of Neutrons in a Steady-state Reactor", Nucl. Sci. Eng. 26, 565 (1966)

(21) J.G. BRUNA et al. - "Etude Preliminaire de la Cinetique d'un Reactor par la Methode des Probabilities", Rapport CEA, France, n. 2454 (1964)

(22) A.J. LINDEMAN, L. RUBY - "Subcritical Reactivity From Neutron Statistics", Nucl. Sci. Eng. 28, 308 (1967)

(23) J.B. DRAGT, E. TURKCAN - "Some Remarks on the Partical Use of the p-Method in Reactor-Noise Analysis", Nukleonik 10, 67 (1967)

(24) N. PACILIO-"Comments on Some Probability Methods in Reactor-Noise Time-Analysis", Annual Report (1967-1968) ANL 7410 (in press)

(25) D. BABALA - "Neutron Counting Statistics in Nuclear Reactors", KR-114 Kjeller Report, Norway (1966)

(26) D.T. AUSTIN et al. - "Comparison of the Waiting-Time alpha with the Rossi-alpha", Trans. Am. Nucl. Soc. 10, 591 (1967)

(27) D. BABALA - "Interval Distribution in Nuclear Counting Statistics", Nucl. Sci. Eng. 28, 243 (1967)

(28) D. BABALA, R. OGRIN - "Measurement of the Prompt Neutron Decay Constant of the NORA Reactor by the Interval Distribution Technique", Nucl. Sci. Eng. 29, 367 (1967)

(29) J.B. DRAGT - "Reactor Noise Analysis by Means Polarity Correlation", Nukleonik 8, 225 (1966)

(30) C.E. COHN - "Fast-Reactor Noise Analysis with an On-line digital Computer", Trars. Am. Nucl. Soc. 10, 285 (1967) 
DISTRIBUTION

(4) Argonne National Laboratory
R. Avery
E. F. Bennett
C. E. Cohn
B. J. Toppel

(3) AEC, RDT Site Representative

W. E. Fry

P. G. Holsted -2

(4) AEC, RL

M. R. Carrothers

H. A. House

C. L. Robinson

C. R. Oualheim

(4) AEC, Washington

D. E. Erb

J. Kruth

M. Shaw

I. F. Zartman

(2) Atlantic Richfield Hanford Company R. D. Carter

File

(1) Babcock and Wilcox

$$
\text { H. M. Jones }
$$

(3) Battelle Memorial Institute

(1) Brookhaven National Laboratory

$$
\text { J. Chernick }
$$

(2) Combustion Engineering.

W. P. Chernock

R. Harding

(14) CNEN-Centro Studi-Nucleaire

Casaccia, Rome, Italy

Ugo Farinelli

A. Gandini

P. Loizzo

R. Martinelli

N. Pacilio (10)

(5) Division of Technical

Information Extension

(for UC-80 Dist)

(2) AEC, Chicago Patent Group

G.H. Lee

R.K. Sharp
(4) Douglas United Nuclear F. Bouse (Tech. Info) (3) File

(1) E. I. duPont de Nemours \& $\frac{\text { Co., Inc., SRL }}{\text { G. Dessauer }}$

(1) General Electric Co., San Jose D. L. Fischer

(1) General Electric Company, Vallecitos Atomic Lab.

(1) Idaho Nuclear Corp. D. deBoisblanc

(1) Nuclear Associates International Corp. J. R. Fisher

(1) Stanford Research Institute, Menlo Park C. Velez

(1) United Nuclear Corp. G. Sofer

(1) University of California Berkeley L. Ruby

(1) University of Florida Gainesville R. E. Uhrig

(2) Westinghouse Electric Corp. H. Graves

F. L. Langford

(57) Battelle-Northwest

F. W. Albaugh

N. E. Carter

D. E. Christensen

E. D. Clayton

E. C, Davis, Jr.

F. G. Dawson

D. E. Deonigi

B. H. Duane 
E. A. Eschbach

J. R. Fishbaugher

F. R. Fisher

J. C. Fox

R. J. Hall

R. E. Heineman

P. L. Hofmann

U. P. Jenquin

R. D. Johnson

J. W. Kutcher

D. D. Lanning

R. C. Liikala

C. W. Lindenmeier

R. P. Matsen

E. T. Merrill

J. H. Nail

D. L. Prezbindowski

W. L. Purcell

J. J. Reginbal

G. W. Riedeman

D. P. Schively

L. C. Schmid

J. R. Sheff

G. L. Simmons

R. I. Smith (10)

D. H. Stewart

W. P. Stinson

D. H. Thomsen

V. 0 . Uotinen

W. P. Walsh

L. D. Williams

N. G. Witt enbrock

$\mathrm{J}$. R. Worden
Tech. Information Files (5)

Technical Publications (2) 